\title{
An Unusual Cause of Generalized Insulin Edema and Truncal Neuropathy
}

\author{
Nicola Tufton a, b, Simon Coppack ${ }^{\mathrm{a}}$, Bobby Huda ${ }^{\mathrm{a}}$
}

\begin{abstract}
Insulin edema and acute neuropathy are rare side effects of insulin initiation or rapid improvement in glycemic control. Both conditions occurring simultaneously are very rare and there are no previous data on truncal neuropathy as a dominant feature. A 35-year-old lady, who presented with an infected, necrotic fourth finger and was admitted for debridement and antibiotics, developed diabetic ketoacidosis. Past medical history included latent autoimmune disease of adults. She was non-compliant with medication and had experienced significant weight loss (37 kg; body mass index (BMI) $15.1 \mathrm{~kg} / \mathrm{m}^{2}$ ). She was glutamic acid decarboxylase antibody positive, with poor glycemic control (HbAlc $133 \mathrm{mmol} / \mathrm{mol}(14.3 \%)$ ). She was treated with intravenous and then subcutaneous insulin. From day 3, she developed significant pitting edema to her umbilicus. She was treated with furosemide $40 \mathrm{mg}$ once daily and fluid restriction. Other causes of edema were excluded. Peak weight gain was $5 \mathrm{~kg}$ and edema resolved 2 weeks later. She also developed a painful sensory neuropathy over dermatomes T12-L2 on day 5, which persisted for several months before resolution. Magnetic resonance imaging was normal and nerve conduction studies showed generalized diabetic sensorimotor neuropathy. This is the first report of edema and truncal neuropathy developing secondary to insulin initiation and a rapid improvement in glycemic control. Our case was particularly susceptible due to poor glycemic control, low bodyweight, hepatic dysfunction and low serum albumin. This case highlights the importance of being aware of the rare complications of insulin therapy, particularly in susceptible patients.
\end{abstract}

Keywords: Type 1 diabetes; Insulin edema; Truncal neuropathy; Painful neuropathy; Insulin side effects

Manuscript accepted for publication October 16, 2015

aDepartment of Diabetes, Royal London Hospital, Barts Health NHS Trust, London, UK

${ }^{b}$ Corresponding Author: Nicola Tufton, Department of Diabetes \& Metabolism, John Harrison House, Royal London Hospital, Barts Health NHS Trust, Whitechapel, London E1 1BB, UK. Email: nicola.tufton1@nhs.net

doi: http://dx.doi.org/10.14740/jmc2345w

\section{Introduction}

Insulin edema and acute neuropathy are rare side effects of insulin initiation or rapid improvement in glycemic control. Both conditions occurring simultaneously in the same individual are very rare and there are no previous data on truncal neuropathy as a dominant feature. Similarities between our case and another recently reported case suggest risk factors for both complications are similar, which may indicate a shared underlying pathophysiological mechanism. Mild insulin edema is a recognized complication of insulin initiation, and probably under reported, but there are very few reported cases of generalized edema.

We report for the first time, a case of generalized edema and truncal neuropathy, occurring simultaneously after insulin initiation, with numerous similarities to another recently reported case of generalized edema and generalized neuropathic pain following initiation of insulin pump.

\section{Case Report}

A 35-year-old lady presented with a painful discolouration of her right fourth finger and was admitted under the plastic surgical team for debridement and washout of her necrotic finger (she refused amputation) and antibiotic treatment for hand cellulitis. In Australia, 5 years previously, she had been diagnosed with diabetes mellitus and subsequently latent autoimmune diabetes (LADA), as she was strongly positive for glutamic acid decarboxylase (GAD) antibody (>2,000,000 units/L). She refused insulin treatment and therefore was managed with gliclazide $80 \mathrm{mg}$ twice daily and lifestyle intervention. She was lost to follow-up after this initial diagnosis. She did not smoke or drink alcohol, and had no relevant family history. Glycemic control was poor, with $\mathrm{HbA} 1 \mathrm{c}$ prior to admission at $133 \mathrm{mmol} /$ mol (14.3\%), and she had lost a significant amount of weight $(18 \mathrm{~kg})$ in the preceding 3 months.

On admission, she was underweight (37.5 kg, BMI 15.1 $\mathrm{kg} / \mathrm{m}^{2}$ ), with a necrotic right fourth digit, but physical examination revealed no other specific abnormalities. She had normal renal and liver function (eGFR $149 \mathrm{~mL} / \mathrm{min}$, albumin $41 \mathrm{~g} / \mathrm{L})$, and raised C-reactive protein $(107 \mathrm{mg} / \mathrm{L})$ and white blood count (WBC $11.8 \times 10^{9} / \mathrm{L}$ ). Arterial blood gas showed acidosis ( $\left.\mathrm{pH} 7.27, \mathrm{HCO}_{3} 13 \mathrm{mEq} / \mathrm{L}\right)$, glucose $24 \mathrm{mmol} / \mathrm{L}$ and 
urinary ketones $4+$. She received standard treatment for diabetic ketoacidosis (DKA) and then converted to a basal-bolus regimen of insulin glargine and aspart with a total daily insulin dose (TDD) of 74 units on day 2.

On day 3, she developed pitting edema to her knees, her lung fields were clear and jugular venous pressure was not raised. Her total protein $(45 \mathrm{~g} / \mathrm{L})$ and albumin $(25 \mathrm{~g} / \mathrm{L})$ had reduced but with otherwise preserved renal/liver function (sodium $141 \mathrm{mmol} / \mathrm{L}$, urea $3.2 \mathrm{mmol} / \mathrm{L}$, creatinine $28 \mu \mathrm{mol} / \mathrm{L}$, bilirubin $3 \mu \mathrm{mol} / \mathrm{L}$, hemoglobin $11.2 \mathrm{~g} / \mathrm{dL}$, hematocrit 0.3 , WBC $5.9 \times 10^{9} / \mathrm{L}$ ). By day 5 , she had significant pitting edema to her umbilicus and had developed a transaminitis (bilirubin $3 \mu \mathrm{mol} / \mathrm{L}$, alanine transaminase $256 \mathrm{U} / \mathrm{L}$, aspartate transaminase $191 \mathrm{U} / \mathrm{L}$, alkaline phosphatase $425 \mathrm{U} / \mathrm{L}$, gamma-glutamyl transpeptidase $987 \mathrm{U} / \mathrm{L})$, which was thought to be secondary to antibiotic therapy and sepsis; it resolved as she clinically improved.

On day 20, she reported painful neuropathic symptoms affecting mainly her trunk. She had marked allodynia over T12L2 dermatomes but with no other neurological signs.

She was extensively investigated for causes of edema and neuropathy. There was no evidence of proteinuria and she had normal thyroid function. An ultrasound demonstrated normal renal/liver architecture with bilateral small pleural effusions and a small amount of free fluid around the liver; a transthoracic echocardiogram was normal. Her B12 (570 ng/L) and folate $(14.4 \mu \mathrm{g} / \mathrm{L})$ levels were normal, as was a vasculitic and virology screen (negative serology for Lyme disease, syphilis, HIV and hepatitis). Nerve conduction studies showed slowing of sensory and motor conduction velocities supporting the diagnosis of diabetic sensorimotor neuropathy. Magnetic resonance imaging (MRI) of her brain and spine was unremarkable.

She was commenced on $40 \mathrm{mg}$ furosemide daily from day 7. Fluid restriction to $1.5 \mathrm{~L}$ daily was enforced from day 9 and reduced further to $750 \mathrm{~mL}$ on day 11 . Her albumin gradually normalized to $35 \mathrm{~g} / \mathrm{L}$ by day 14 . Her peak weight gain was 5 $\mathrm{kg} 20$ days after her admission. She was discharged on a basalbolus insulin regimen of glargine 24 units and novorapid 10 units with each meal.

With a tentative diagnosis of insulin-induced edema, diuretics and fluid restriction were tapered off and edema gradually settled 2 weeks after discharge.

No other cause was found for her neurology, and the truncal allodynia eventually settled spontaneously 3 months after discharge.

\section{Discussion}

The true incidence of insulin edema is unknown and is probably under reported [1]. A review of the edematogenic properties of insulin describes clinical risk factors that increase the risk of developing edema. These include being aged $20-40$ years, type 1 diabetes, poor glycemic control (including both hypoglycaemia episodes and following treatment of DKA), poor nutritional status, low body weight, new-onset diabetes and higher doses of insulin therapy [1]. Our case met almost all of these high risk criteria as did another recently reported similar case, of generalized edema and neuropathy occurring after initiation of insulin pump therapy [2]. In that case the acute neuropathy was generalized, rather than the rarer truncal distribution.

The mechanisms of insulin edema are unknown [1], and several theories have been postulated. These include increased microvascular permeability [3], direct triggering of vascular endothelial growth factors [4], as well as a direct action of insulin on the renal tubules causing anti-natriuresis [5]. Associations with malnutrition may be secondary to unmasking of thiamine deficiency by insulin, and lactate and pyruvate accumulation produce peripheral vasodilation and edema [1]. There is also a proposed genetic predisposition with the 3243 mitochondrial tRNA ${ }^{\text {Leu(UUR) }}$ mutation [6].

Takahiro et al suggest a link between hepatic dysfunction and a predisposition to developing insulin edema [5]. Acute hepatic decompensation is associated with transient insulin resistance and hyperinsulinemia, due to impaired glucose utilization in peripheral tissue and decreased insulin degradation and clearance. Once established, both refractory hyperglycemia and body water retention are promoted by intensifying sodium reabsorption in the renal tubules, increasing vascular permeability causing hypoalbuminemia and therefore reducing oncotic pressure [5]. It is possible that this contributed to the development of insulin-induced edema in our patient and transient derangement of liver function has been reported in other cases $[2,7]$.

Many resolve with conservative management along with reduction in insulin dosing or dietary salt restriction $[1,2,7$, 8]. If there is evidence of malnutrition, thiamine supplementation should be initiated [1]. Diuretic use with furosemide 40 $\mathrm{mg}$ once daily, bumetanide $1 \mathrm{mg}$ per day or spironolactone 100 mg per day have been tried with variable success $[1,2,9,10]$. There have been a few reported successfully treated cases of initiating vasoactive drugs, such as ephedrine $15 \mathrm{mg}$ per day divided over three doses, in severe edema resistant to diuretics $[1,9,10]$.

Treatment-induced neuropathy is characterized by a reversible acute, severe painful, peripheral nerve degeneration and autonomic dysfunction after intensive glycemic control [11], which can occur at proximal sites including the trunk [12]. There is very little literature on acute truncal neuropathy. An older case series reported that symptoms were mainly sensory, asymmetric, occurring in a root distribution with an equal sex distribution and good prognosis. Most spontaneously recovered over 3 months [8].

There have been reported associations between acute severe neuropathy and significant weight loss, both intentionally (dieting or withholding of insulin) and unintentionally [11]; this was particularly relevant in our patient.

\section{Conclusion}

Both insulin-induced edema and acute painful neuropathy are recognized, albeit rare side effects of initiation or increasing insulin therapy, with resultant rapid correction of glycemic 
control. There are numerous similarities between our case and one previously reported [2], both with regard to risk factors (similar preceding time course of diabetes, no underlying cardiac, renal or hepatic disease, poor glycemic control demonstrated by high $\mathrm{HbA1c}$, underweight) and clinical course of disease. Whilst rare these two side effects occurring simultaneously, suggest a possible similar underlying pathophysiology.

\section{Funding}

This research did not receive any specific grant from any funding agency in the public, commercial or not-for-profit sector.

\section{Declaration}

The authors have none to declare.

\section{References}

1. Kalambokis GN, Tsatsoulis AA, Tsianos EV. The edematogenic properties of insulin. Am J Kidney Dis. 2004;44(4):575-590.

2. Rothacker KM, Kaye J. Insulin oedema and treatment-induced neuropathy occurring in a 20 -year-old patient with Type 1 diabetes commenced on an insulin pump. Diabet Med. 2014;31(1):e6-e10.

3. Jaap AJ, Shore AC, Gartside IB, Gamble J, Tooke JE. Increased microvascular fluid permeability in young type 1 (insulin-dependent) diabetic patients. Diabetologia. 1993;36(7):648-652.

4. Poulaki V, Qin W, Joussen AM, Hurlbut P, Wiegand SJ,
Rudge J, Yancopoulos GD, et al. Acute intensive insulin therapy exacerbates diabetic blood-retinal barrier breakdown via hypoxia-inducible factor-1alpha and VEGF. J Clin Invest. 2002;109(6):805-815.

5. Zendaa T, Murasea Y, Yoshidaa M, Muramotoa M, Okad$\mathrm{ab} \mathrm{T}$, Yagic K. Does the use of insulin in a patient with liver dysfunction increase water retention in the body, i.e. cause insulin oedema? Eur J of Gastroenterology \& Hepatology. 2003;15(5):545-549.

6. Suzuki Y, Kadowaki H, Taniyama M, Kadowaki T, Katagiri H, Oka Y, Atsumi Y, et al. Insulin edema in diabetes mellitus associated with the 3243 mitochondrial tRNA(Leu(UUR)) mutation; case reports. Diabetes Res Clin Pract. 1995;29(2):137-142.

7. Lee P, Kinsella J, Borkman M, Carter J. Bilateral pleural effusions, ascites, and facial and peripheral oedema in a 19-year-old woman 2 weeks following commencement of insulin lispro and detemir--an unusual presentation of insulin oedema. Diabet Med. 2007;24(11):1282-1285.

8. Ellenberg M. Diabetic truncal mononeuropathy--a new clinical syndrome. Diabetes Care. 1978;1(1):10-13.

9. Kalambokis and O'Neal. Insulin oedema in CF and Hopkins, insulin-induced oedema using ephedrine. Diabetes Care. 2012;35.

10. Hopkins DF, Cotton SJ, Williams G. Effective treatment of insulin-induced edema using ephedrine. Diabetes Care. 1993;16(7):1026-1028.

11. Gibbons $\mathrm{CH}$, Freeman R. Treatment-induced diabetic neuropathy: a reversible painful autonomic neuropathy. Ann Neurol. 2010;67(4):534-541.

12. Dabby R, Sadeh M, Lampl Y, Gilad R, Watemberg N. Acute painful neuropathy induced by rapid correction of serum glucose levels in diabetic patients. Biomed Pharmacother. 2009;63(10):707-709. 